\title{
Investigation of the Influence of PVD Coatings Deposited on HSS Milling Cutter
}

\author{
Ales Jaros, Zdenek Fiala \\ Department of Machining Technology, Institute of Manufacturing Technology, Faculty of Mechanical Engineering, Brno \\ University of Technology, Technicka 2896/2, Brno 616 69, Czech Republic. \\ E-mail: jaros.a@stud.fme.vutbr.cz, fiala.z@fme.vutbr.cz
}

This article deals with the benefits of PVD coatings ((Al,Ti)N; (Al,Ti,Cr)N and nanocomposite coating nACoß) applied to HSS three edges end milling cutters (producer ZPS - Frezovaci nastroje, Zlin, CZ). The coatings were synthesized by a cathodic-arc deposition process (producer Liss, Roznov pod Radhostem, CZ). Machining was carried out on the vertical milling machine FB $32 \mathrm{~V}$ with using process liquid. Set up cutting conditions were constant throughout the machining. The aim of this experiment was to compare coated and uncoated HSS end milling cutters and find out the benefits of three kinds of PVD coatings. The monitored parameters were force loading and flank wear. Piezoeletrical dynamometer Kistler 9257B was used for measuring force loading and workshop optical microscope was used for measuring flank wear (criterion VB). The construction steel C45E (1.1191; CSN 41 2050) was used as workpiece material. Best results were achieved by tool with PVD coating (Al,TiCr)N.

Keywords: PVD coating, High-speed steel, milling, force loading, flank wear

\section{Acknowledgement}

This research work was supported by the BUT, Faculty of Mechanical Engineering, Brno, Specific research 2016, with the grant "Research of modern production technologies for specific applications", FSI-S-16-3717.

\section{References}

[1] HUMÁR, A. (2008). Materiály pro řezné nástroje. Praha: MM publishing, 235 s. ISBN 978-80-254-2250-2.

[2] JAROŠ, A., KOLÁŘ, L., FIALA, Z. (2014). The development of new HSS milling cutter with negative geometry for roughing operations. ERIN 214, 8th International Conference for Young Researchers and Ph.D. Students. 1. Litera Brno: Brno University of Technology, s. 1-8. ISBN: 978-80-214-4931- 2.

[3] KOVALEV, A.I, G.S FOX-RABINOVICH, D.L WAINSTEIN a V.P MISHINA. (2000). Studying the structure of films generated on HSS-based deformed powder materials cutting tool surface. Wear. roč. 238, č. 2, s. 81-92. ISSN 00431648. DOI: 10.1016/S0043-1648(99)00301-4.

[4] CHANG, Yin-Yu, Da-Yung WANG, Chi-Yung HUNG. (2005). Structural and mechanical properties of nanolayered TiAlN/CrN coatings synthesized by a cathodic arc deposition process. Surface and Coatings Technology. roč. 200, 5-6, s. 1702-1708. ISSN 02578972. DOI: 10.1016/j.surfcoat.2005.08.088.

[5] MATTOX, D. (2010). Handbook of physical vapor deposition (PVD) processing: principles, technology, and applications. 2nd ed. Amsterdam: Elsevier, xlvi, 746 s. ISBN 978-0-81-552037-5.

[6] XU, J., GU, L., LUO, S. (2014). Dynamic Analysis for High-speed Cutters of Five-axis CNC Milling Machine. In: Manufacturing Technology, Vol. 14, pp. 643-650. ISSN 1213-2489.

[7] FIALA, Z., JAROŠ, A., SEDLÁK, J., KOLÁŘ, L., BLAŽKOVÁ, V. (2015). Effect of Spindle Unit Extrusion on Stability of Machining Process. Manufacturing Technology, roč. 15, č. 3, s. 329-333. ISSN: 1213- 2489.

[8] OSIČKA, K., FIŠEROVÁ, Z., OTOUPALÍK, J. (2015). Influence of cutting tool overhangs at machining of hardened steels. Manufacturing Technology, roč. 15, č. 2, s. 188-191. ISSN: 1213- 2489.

[9] JAROŠ, A., FIALA, Z., DVOŘÁČEK, J. (2012). Investigation of the influence of AlTiCrN coating for dry milling of construction steel 1.8159. In Sborník př́spěvků Mezinárodní Masarykovy konference pro doktorandy a mladé vědecké pracovníky 2012. 1. Hradec Králové: MAGNANIMITAS, s. 3475-3482. ISBN: 978-80-905243-3- 0.

[10] FIALA, Z., JAROŠ, A., SEDLÁK, J., KOLÁŘ, L., BLAŽKOVÁ, V. (2015). Effect of Spindle Unit Extrusion on Stability of Machining Process. Manufacturing Technology, roč. 15, č. 3, s. 329-333. ISSN: 1213- 2489.

[11] Tested HSS end milling cutters. [Online]. [Visited $1^{\text {st }}$ November 2015]. Available at: http://www.zps-fn.cz/.

[12] Deposited PVD coatings on HSS end milling cutters. [Online]. [Visited $3^{\text {rd }}$ November 2015]. Available at: http:// http://www.liss.cz/nabidka.php?podskupina $=4$.

[13] Chemical properties of construction steel C45. [Online]. [Visited $4^{\text {rd }}$ November 2015]. Available at: http:// http://www.bolzano.cz/cz/technicka-podpora/technicka-prirucka/tycove-oceli-uhlikove-konstrukcni-a-legovane/oceli-k-zuslechtovani-podle-en-10083-1/prehled-vlastnosti-oceli-c45. 